\title{
Contrast-Enhanced Mammogram: A New Game-Changer in Breast Imaging. Indications with Case-Based Experience
}

\author{
Chotai Niketa $^{1^{*}(\mathbb{D})}$, Loke Siu Cheng ${ }^{2}$, Tan Ern $\mathrm{Yu}^{3}$ \\ ${ }^{1}$ RadLink Diagnostic Imaging Center, Visiting Consultant, Department of Radiology, Tan Tock Seng Hospital, Singapore \\ ${ }^{2}$ Department of Diagnostic Imaging, Tan Tock Seng Hospital, Singapore \\ ${ }^{3}$ Department of Surgery, Tan Tock Seng Hospital, Singapore \\ Email: ${ }^{\star}$ niketachotai@gmail.com
}

How to cite this paper: Niketa, C., Cheng, L.S. and Yu, T.E. (2021) Contrast-Enhanced Mammogram: A New Game-Changer in Breast Imaging. Indications with Case-Based Experience. Advances in Breast Cancer Research, 10, 184-199.

https://doi.org/10.4236/abcr.2021.104016

Received: August 20, 2021

Accepted: October 5, 2021

Published: October 8, 2021

Copyright () 2021 by author(s) and Scientific Research Publishing Inc. This work is licensed under the Creative Commons Attribution-NonCommercial International License (CC BY-NC 4.0). http://creativecommons.org/licenses/by-nc/4.0/

\begin{abstract}
Mammograms are the mainstay of diagnostic breast imaging and cancer screening. Despite advances in technology such as Full Field Digital Mammography (FFDM) and Digital Breast Tomosynthesis (DBT), these imaging techniques are purely structural, and are unable to overcome shortcomings in mammography posed by dense breast parenchyma. Magnetic Resonance Imaging (MRI) is the most sensitive modality in breast imaging, due to its functional aspects in addition to structural imaging with this modality. The use of MRI is however constrained by cost and availability. The utilization of intravenous contrast with mammograms introduces a functional element to imaging. This greatly improves the sensitivity of the examination, approaching sensitivity levels of Magnetic Resonance Imaging (MRI) in the detection of primary breast cancer. With increased sensitivity afforded by assessment of tumor neo-vascularity, as well as its low cost, low energy imaging that is more readily available than MRI, Contrast-Enhanced Mammography (CEM) if more readily available than MRI, this imaging modality is a potential game-changer in breast imaging. In this article, we share our experience in the use of CEM, for indications previously reserved for MRI, with the literature review of these indications. In resonance with prior studies, we echo the ease of performing and reporting of CEM as well as greater patient comfort as the distinct advantages of CEM. In spite of slightly higher radiation dose and some risks related to contrast media, functional results at a significantly lower cost may change how we practice breast imaging in the future using CEM.
\end{abstract}

\section{Keywords}

Contrast-Enhanced Mammography, Clinical Use, Advantages, Indications 


\section{Introduction}

A mammogram is the most frequently used modality for diagnostic breast imaging and cancer screening [1] [2], in no small part to its widespread availability, short examination time, reproducibility, ease of interpretation and ability to detect microcalcifications. Dense breast parenchyma is a major limiting factor that reduces the sensitivity of the mammogram by nearly half in extremely dense breast tissue [3] [4]. Though tomosynthesis [5] partly overcomes this limitation by reducing tissue overlap to some degree, these techniques remain purely morphology-based.

By contrast, breast Magnetic Resonance Imaging (MRI) is highly sensitive, with a functional element of imaging using intravenous contrast, in addition to its superior soft-tissue contrast and three-dimensional format; this allows for an examination independent of breast density [6]. The use of intravenous contrast leverages in imaging characteristics of tumor neo-angiogenesis, and significantly increases sensitivity in breast cancer detection [7]. In addition to its anatomical advantage through three-dimensional visualization, there is better coverage of the chest wall, axilla, and internal mammary nodes. Despite its high sensitivity, routine use of MRI in clinical practice is restricted by issues of accessibility (financial costs, long waiting times), technical performance (long scan time, difficulties in patients with claustrophobia) as well as diagnosis (high-false positive rates, inability to detect microcalcifications).

Contrast-Enhanced Mammogram (CEM) aims to address the shortcomings of conventional mammography, by introducing an element of functional imaging, similar to breast MRI.

Dual-energy mammogram exposures are obtained after intravenous injection of iodine-based contrast agent. The low-energy exposure produces an image that is similar to a conventional Full-Field Digital Mammogram (FFDM), while the recombined image obtained from the subtraction of two images provides details of contrast enhancement in the breast. It does not require substantial training for technologists already involved in mammography and is easy to perform. For the radiologist, there is no significant departure from interpretation in conventional mammography. The additional recombined images allow for detection of lesions that show contrast enhancement which indicates increased vascularity, which may suggest underlying neo-angiogenesis such as in breast cancer.

Studies have already shown CEM to have higher sensitivity than mammograms, tomosynthesis, or even a combination of mammograms and ultrasound [8] [9]. In fact, many studies have shown CEM results to be nearly comparable to Dynamic Contrast-Enhanced MRI (DCE-MRI), which is encouraging.

This article aims to share our initial clinical experience of using CEM. Indications for performing breast MRI were similarly applied to CEM, given CEM's high sensitivity being similar to breast MRI, allowing for contrast and comparison and to illustrate its usefulness in clinical practice.

The clinical indications are:

- To assess the extent of breast cancer or for pre-operative cancer staging; 
- To monitor the response to neo-adjuvant chemotherapy;

- To look for residual or recurrent breast cancer;

- Surveillance in women with a personal history of breast cancer;

- Problem-solving in equivocal findings on conventional imaging;

- Screening in high-risk women.

We share our experience with cases of CEM used for individual indication instead of MRI, followed by indication-specific literature review and discussion.

\section{Discussion}

\subsection{To Assess the Extent of Breast Cancer or for Pre-Operative Cancer Staging}

MRI is well-established as a modality to assess loco-regional extent of breast cancer pre-operatively. This is especially useful in young women, women with dense breasts, invasive lobular carcinoma and Locally Advanced Breast Cancer (LABC). Accurate assessment of disease extent is an important determining factor in the decision for Breast Conserving Surgery (BCS) versus mastectomy. In LABC, involvement of chest wall and internal mammary nodes are better assessed with MRI, the presence of which may influence the management.

Case 1: A 42-year-old woman presented with a palpable left breast lump. Mammogram (Figure 1(A)) showed extremely dense breast parenchyma (ACR category D) with a suspicious mass in the left breast at 12 o'clock. Ultrasound (Figure 1(B)) confirmed a corresponding BI-RADS 5 mass at 12 o'clock and additionally a smaller indeterminate lesion at 11 o'clock in the same breast, raising possibility of multifocal cancer. Pre-operative CEM was offered for better assessment of disease extent and to rule out synchronous right breast tumor. On CEM (Figure 1(C)), intensely enhancing masses were seen in left breast at 11 and 12 o'clock, correlating with ultrasound findings. Additionally, moderately enhancing non-mass enhancement was identified at 2 o'clock of the same breast. Targeted second look ultrasound (Figure 1(D)) of left breast 2 o'clock region identified an ill-defined mixed echogenic lesion that was also biopsied. Histology from 12 o'clock lesion was reported as a Grade 3 invasive ductal carcinoma while the 2 o'clock lesion was reported as intermediate grade DCIS. This confirmed the disease to be multicentric, changing the overall disease extent, treatment plan as well as prognosis. No enhancing lesions were noted in the contralateral breast on CEM and this was reassuring of absent synchronous tumor.

Case 2: A 48-year-old woman presented with left breast palpable mass; clinically worrisome for breast cancer. Direct CEM was performed for this patient. Low energy images (Figure 2(A)), similar to a regular mammogram, showed a high density, irregular mass in the upper outer quadrant of the left breast. No other lesion was identified in rest of breast on mammogram. Contrast enhanced images (Figure 2(B)) showed a single, avidly enhancing mass in the upper outer quadrant of the left breast at mid third depth. No other concerning enhancement 


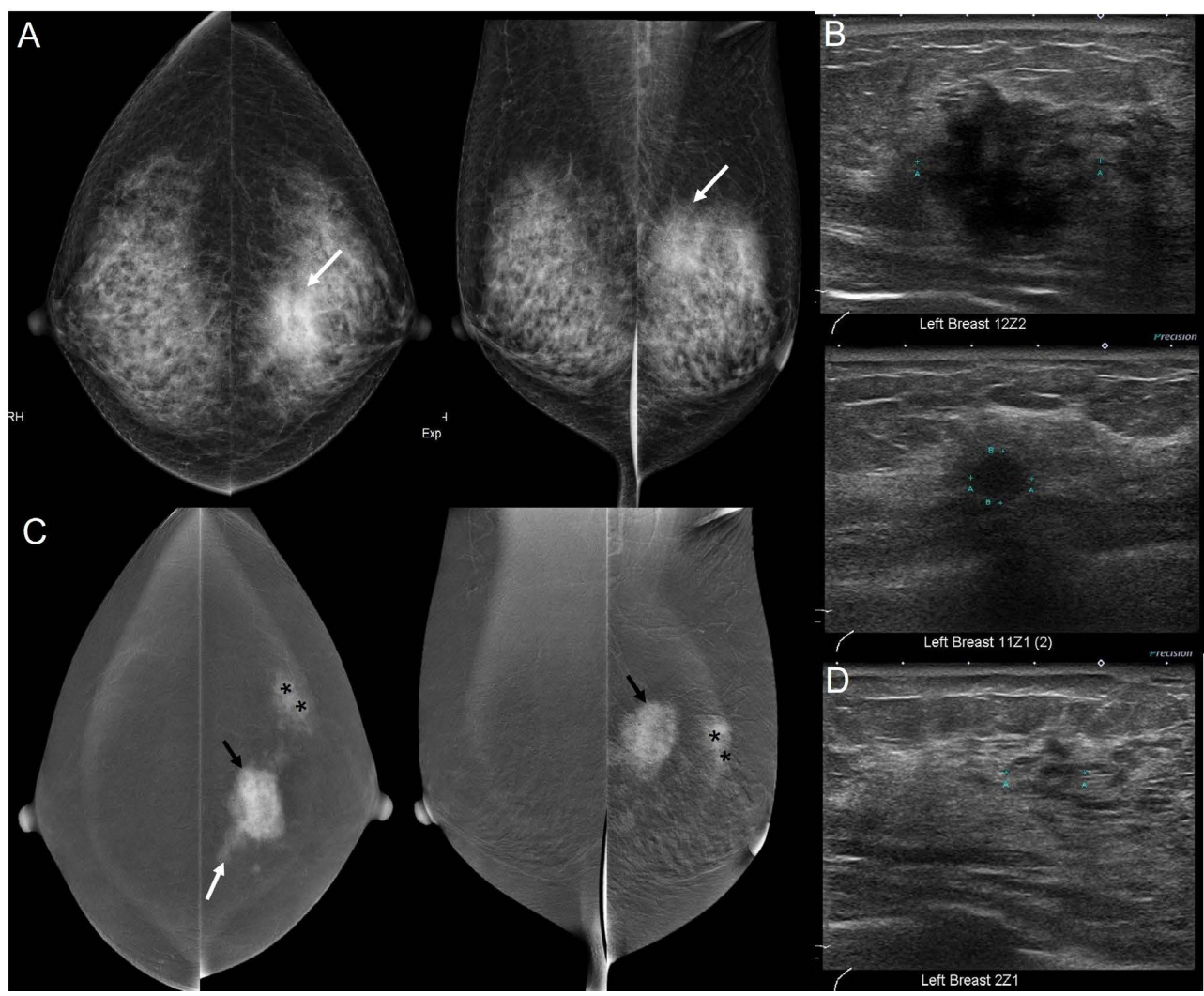

Figure 1. 42-year woman with palpable left breast lump. (A) Standard mammogram shows a high density, irregular mass in the upper central aspect of left breast at 12 o'clock (arrows); (B) Ultrasound shows two irregular hypoechoic masses in the left breast at 12 and 11 o'clock locations; (C) Contrast Enhanced Mammogram shows dominant avidly enhancing mass in the left breast at 12 o'clock (black solid arrow) and a smaller enhancing mass in the left breast at 11 o'clock (white solid arrow). There is also non-mass enhancement seen in the upper outer quadrant of the left breast (black dashed arrow); (D) Second look ultrasound shows a vague, heterogeneous area in the left breast at 2 o'clock to correlate with non-mass enhancement seen on CEM. This was proven to be DCIS on histology.

was seen in the left breast suggesting this to be a unifocal tumor. Contralateral breast did not show any synchronous tumor. Targeted ultrasound of left breast (Figure 2(C)) showed a suspicious correlating mass at the 2 o'clock location, which was later biopsy proven as Grade 2 invasive ductal carcinoma. By using direct CEM, we could get mammographic details as well as make better assessment of disease extent at the first instance that would facilitate quicker treatment planning.

\section{Literature review}

Stephanie A Lee-Felker et al. [10] compared the diagnostic performances of CEM and breast MR imaging in assessing the extent of disease in women with newly diagnosed breast cancer. In their study, CEM showed sensitivity close to MRI (94\% vs 99\%) in detecting primary breast cancer, a significantly higher PPV ( $93 \%$ vs $60 \%$ ), and fewer false-positive results compared to MR imaging (5 vs 45 ) ( $\mathrm{P}<0.001$ for all results). Yun-Chung Cheung et al. [11] in their study on 51 primary cancers showed $88 \%$ sensitivity on mammogram versus $100 \%$ on CEM 


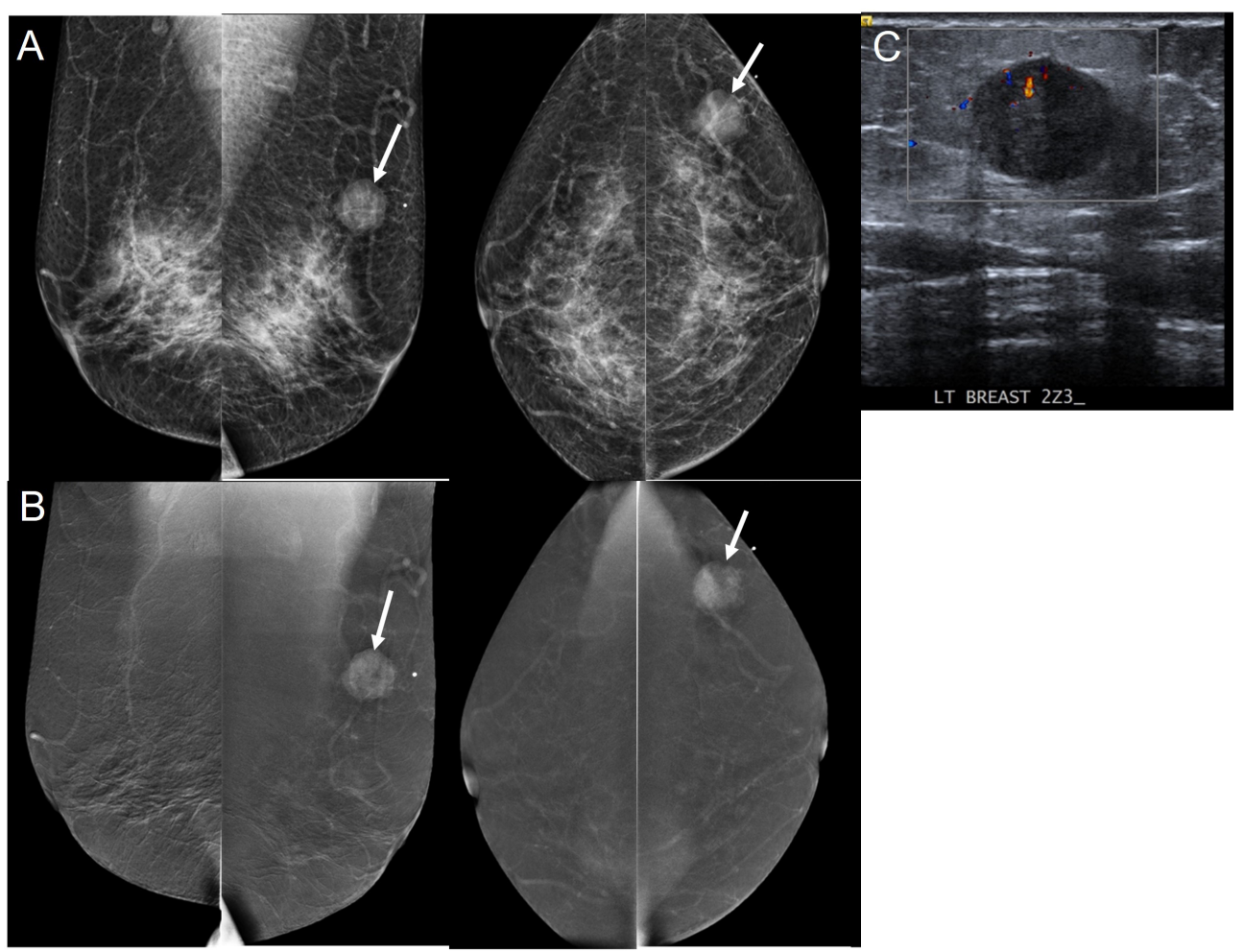

Figure 2. 48-year woman with palpable left breast lump. (A) Low energy images (equivalent to FFDM) show dense breast tissue. There is an irregular, high density mass noted in the upper outer quadrant of the left breast (arrows) underneath the palpable concern (BB marker); (B) Contrast enhanced mammogram images show a single, avidly enhancing mass (arrows) in the left breast suggesting this to be a unifocal tumor. No synchronous right breast cancer is noted; (C) Ultrasound showed a circumscribed hypoechoic mass with microlobulated margins, posterior enhancement and internal vascularity.

and MRI. The tumor size also better correlated with CEM and MRI. Multiple other studies comparing CEM to MRI have also reported equivalent sensitivity of CEM in detecting primary breast cancer with lower false positive rate on CEM [12] [13] [14] [15] [16].

Hobbs et al. [17] in their prospective trial on 49 cases compared patient preference on CEM versus MRI for pre-operative staging. Patients reportedly preferred the experience of CEM to MRI, adding support for the role of CEM as a possible alternative to MRI for breast cancer staging.

Thus, CEM appears to be a promising alternative pre-operative assessment tool for breast cancer patients with dense breasts and/or limited access or contraindications to MRI. At our center, we try to offer direct CEM, rather than FFDM, and targeted ultrasound to patients with high clinical suspicion of breast cancer. This allows for a quicker and more accurate estimation of tumor extent, with reduced cost and radiation to the patient.

\subsection{To Monitor the Response to Neo-Adjuvant Chemotherapy}

Recently, use of Neo-Adjuvant Chemotherapy (NAC) is expanding in clinical practice, not only for locally advanced breast cancer but also in smaller triple 
negative and HER2-positive cancers. The pathological response to NAC has prognostic significance and influences the subsequent surgical approach. Response to NAC is assessed by clinical examination and imaging. MRI has been shown to be the most sensitive imaging modality, at present, to monitor response to NAC. Due to limitations like financial constraint, resource availability and claustrophobia, MRI may not be suitable in all patients post-NAC. CEM may be an alternative in this instance.

Case 1: A 60-year-old woman with a palpable left breast invasive ductal carcinoma with positive left axillary lymphadenopathy underwent CEM pre and post-NAC. Baseline CEM (Figure 3(A) showed avidly enhancing left breast tumor with internal heterogeneity and enlarged metastatic left axillary node. Post-NAC CEM (Figure 3(B)) was performed to assess the suitability for breast conservation. The primary tumor in the left breast showed significant regression of enhancement. A tissue marker clip was seen in the tumor bed. Imaging features were deemed to indicate complete radiological response to NAC. The left axillary node had also regressed. On final surgical histopathology, a $2 \mathrm{~mm}$ residual focus of invasive ductal carcinoma was noted. The axillary nodes were negative for malignancy on final histology.

Case 2: Another 46-year-old woman diagnosed with multicentric right breast tumor with metastatic right axillary adenopathy (Figure 3(C) and Figure 3(D))

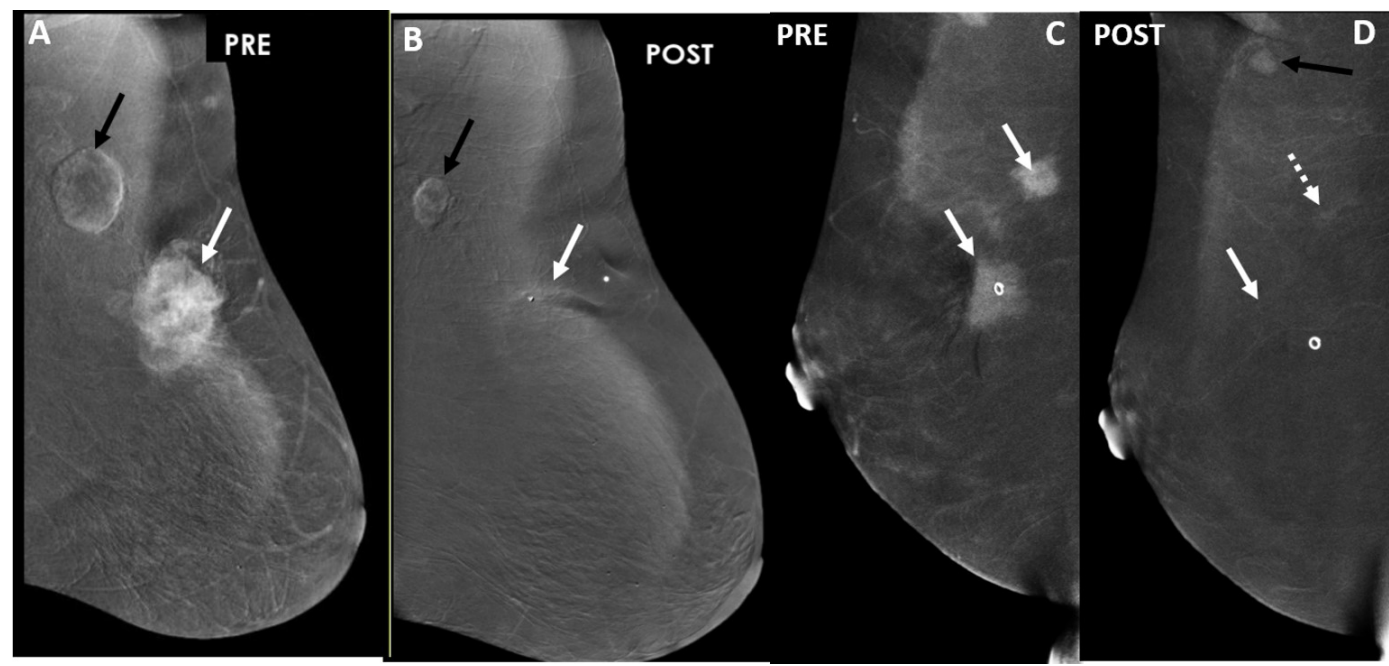

Figure 3. 60-year-old woman with recently diagnosed Grade 3 invasive ductal carcinoma with metastatic left axillary lymphadenopathy. (A) Pre-Neo-adjuvant Chemotherapy (NAC) CEM shows a heterogeneously enhancing mass in the upper outer quadrant of the left breast (white arrow) with enlarged left axillary node (black arrow); (B) Post NAC CEM shows no enhancement in the tumor bed suggesting complete imaging response of tumor to NAC. A small marker clip (white arrow) is noted in the tumor bed. Left axillary node has also significantly resolved (black arrow). 46-year-old woman diagnosed with multicentric right breast cancer, ER/PR as well as HER2 positive; (C) Pre NAC CEM showed two avidly enhancing masses in the upper outer quadrant of the right breast (white arrows) with overlying skin retraction; (D) Post NAC CEM shows a marker clip in one of the tumor bed with no residual enhancement (solid white arrow). There is faint small area of enhancement noted in the second tumor bed located supero-posteriorly (dashed white arrow). The axillary node was also seen enlarged and enhancing (black arrow). Final surgical histopathology confirmed residual $16 \mathrm{~mm}$, grade 3 IDC along with residual metastatic axillary node. 
showed small area of faint residual enhancement in the tumor bed on post-NAC CEM. The axillary node was also seen enhancing. Final surgical histopathology confirmed residual $16 \mathrm{~mm}$, grade 3 IDC along with residual metastatic axillary node.

\section{Literature review}

At present, MRI has been proven to be the most sensitive imaging modality for detecting residual disease, post NAC [18]. Neo-adjuvant chemotherapy has become the standard of care in advanced breast cancers as it facilitates down-staging and increases the likelihood for successful breast conservation. It is also a good tool to assess in-situ chemosensitivity and hence to predict treatment outcomes and prognosis.

In their prospective study on 33 breast cancer patients, Filipe et al. [19] compared CEM and MRI with surgical histopathology in post-NAC patients. While sensitivity of CEM in detecting residual disease was lower compared to MRI (76\% Vs 92\%), the specificity and negative predictive value of CEM were higher compared to MRI ( $87.5 \%$ vs $75 \%$ and $86.4 \%$ vs $75 \%$, respectively). The concordance coefficients between measurements of residual tumor obtained with CEM and MRI as compared to surgical pathology were 0.7 and 0.4 , respectively. Similarly, Pearson correlation coefficient was higher for CEM (0.8) versus MRI (0.5). Lotti et al. [20] reported that the residual tumor size was underestimated on both modalities (mean $4.1 \mathrm{~mm}$ in CESM, $7.5 \mathrm{~mm}$ in MRI). Also, in patients with Complete Response (CR), CEM had higher sensitivity and specificity (100\% and $84 \%$, respectively) compared to MRI ( $87 \%$ and $60 \%$, respectively).

\subsection{To Look for Residual or Recurrent Breast Cancer}

In patients treated with breast conservation therapy, any new finding may raise concern of a recurrence. Changes such as post-operative seroma, fat necrosis, skin thickening, architectural distortion and dystrophic calcifications, may pose a diagnostic dilemma on conventional imaging. Differentiating benign post-therapeutic change versus recurrence is clinically important, but may prove challenging in some cases, requiring biopsy for a definitive answer. In these cases, breast MRI may be used as a problem-solving tool; CEM might be seen as a potential alternative.

Case 1: A 46-year-old woman with previous right breast wide local excision for hormone positive, HER2 negative breast cancer 7 years ago presented with a new palpable lump, adjacent to the scar. Ultrasound (Figure 4(A)) showed a 2.6 $\mathrm{cm}$ mixed echogenic lesion with indeterminate features corresponding to the palpable lump. Mammogram showed dense breast parenchyma without any suspicious features. Tissue diagnosis was suggested for the patient to rule out a recurrence. Pre-biopsy CEM revealed no contrast enhancement in the region of the palpable concern (Figure 4(B)). Histology performed later confirmed the lesion to be post-operative fibrosis with benign breast tissue.

Case 2: A 52-year-old woman with a similar history of a new palpable lump in 


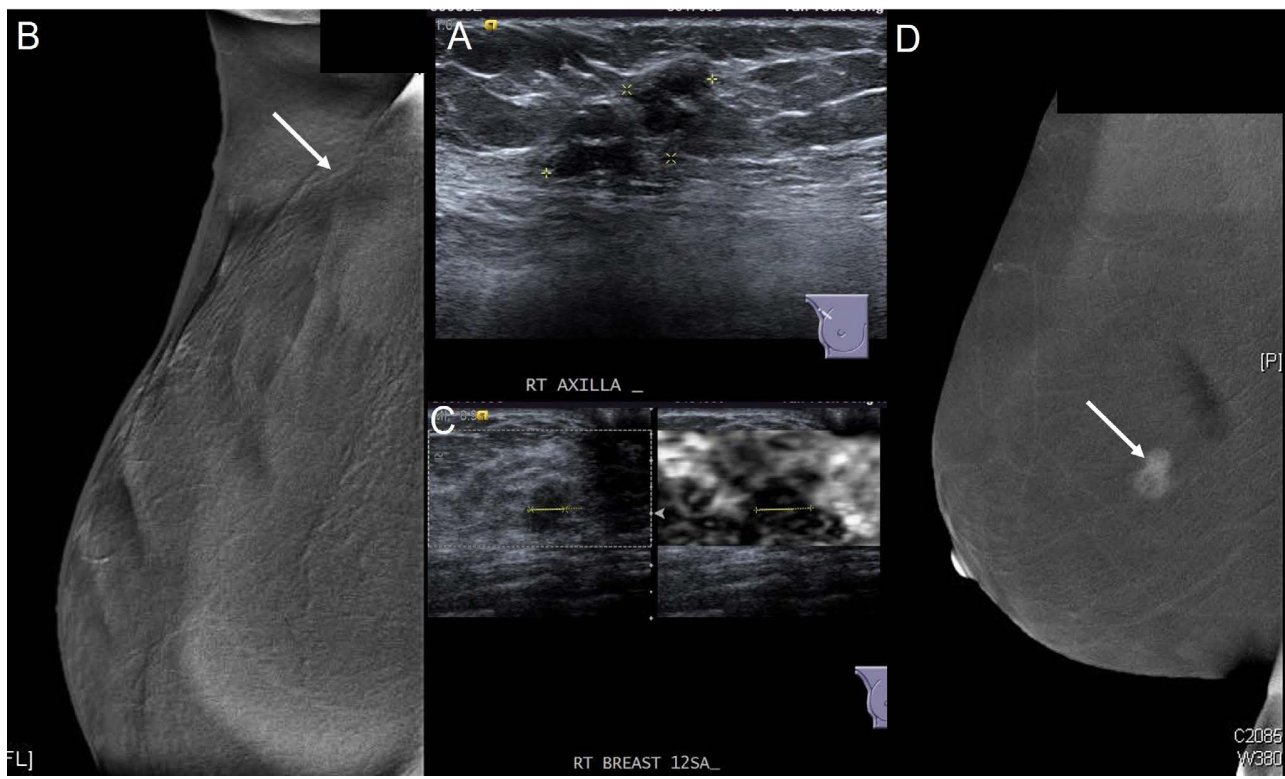

Figure 4. 46-year woman with right breast wide excision done 7 years ago, now presenting with new palpable lump in the region of scar. (A) Ultrasound shows a mixed echogenic lesion (arrow) in the region of palpable concern; (B) Contrast enhanced mammogram image shows no suspicious enhancement in the surgical bed (arrow). The lesion was proven to be benign on histology. A 52 -year woman with right breast wide excision done 6 years ago, now presenting with new palpable lump in the region of scar; (C) Ultrasound shows an irregular, hypoechoic mass (arrow) to correlate with the palpable concern; (D) Contrast enhanced mammogram image shows an avidly enhancing mass in the surgical bed (arrow). The lesion was proven to be recurrent IDC.

the region of a previous wide excision scar was referred to the imaging suite for further assessment. The patient was operated 6 years ago for hormone-positive, HER2 negative, grade 2 Invasive ductal carcinoma with metastatic axillary adenopathy. Ultrasound (Figure 4(C)) revealed an indeterminate hypoechoic lesion underneath the palpable concern. Initial biopsy was reported as benign breast tissue with post-operative changes. Radiology-pathology concordance was questioned. Contrast enhanced mammogram was then performed that showed the lesion to enhance avidly (Figure 4(D)). This was considered a finding of concern and histology was deemed discordant. A repeat ultrasound guided biopsy was carried out, which confirmed invasive ductal carcinoma recurrence. In this case, findings on CEM bolstered initial imaging suspicions which were discordant on pathology, making the case for repeat biopsy and subsequent proof of disease recurrence.

\section{Literature review}

At present there is limited literature on the use of CEM in the diagnosis of recurrence and further research is warranted. Helal and Mansour et al. [21] assessed the role of CEM in detecting residual disease in post-operative breast of cancer patients. CEM enhanced the sensitivity of detecting breast cancer from $50 \%$ to $91.17 \%$ and increased accuracy from $37 \%$ to $82 \%$ as compared to conventional mammogram. However, the data remains limited in this context and needs further evaluation. 


\subsection{Surveillance in Patients with Personal History of Breast Cancer}

Despite advancements in breast cancer treatment, disease recurrence after successful initial treatment remains a real concern. Annual mammography is currently the gold standard for surveillance in post-operative breast [22]. Additional ultrasound and MRI may be performed in cases of suspicious findings on conventional imaging. In their recent recommendations, the American College of Radiologists (ACR) have included adjuvant annual MRI surveillance for patients with a personal history of breast cancer at pre-menopausal age, especially if associated with dense breasts or other high-risk factors like positive family history. This may however not be routinely practiced at many places, due to limitations such as high cost and high false positive results. CEM may be considered as an alternative to breast MRI in such cases.

Case 1: A 64-year-old woman with previous personal history of right mastectomy for breast cancer was detected to have a new $5 \mathrm{~mm}$ group of punctate microcalcifications in the left breast on routine surveillance mammogram (Figure 5(A)). On ultrasound, a $5 \mathrm{~mm}$ indeterminate hypoechoic lesion with internal microcalcifications (Figure 5(B)) was identified to correlate with the mammographic abnormality. Pre-biopsy CEM (Figure 5(C)) showed a $16 \mathrm{~mm}$ area of non-mass enhancement in the region of grouped microcalcifications. The extent of enhancement was larger on CEM compared to conventional imaging.
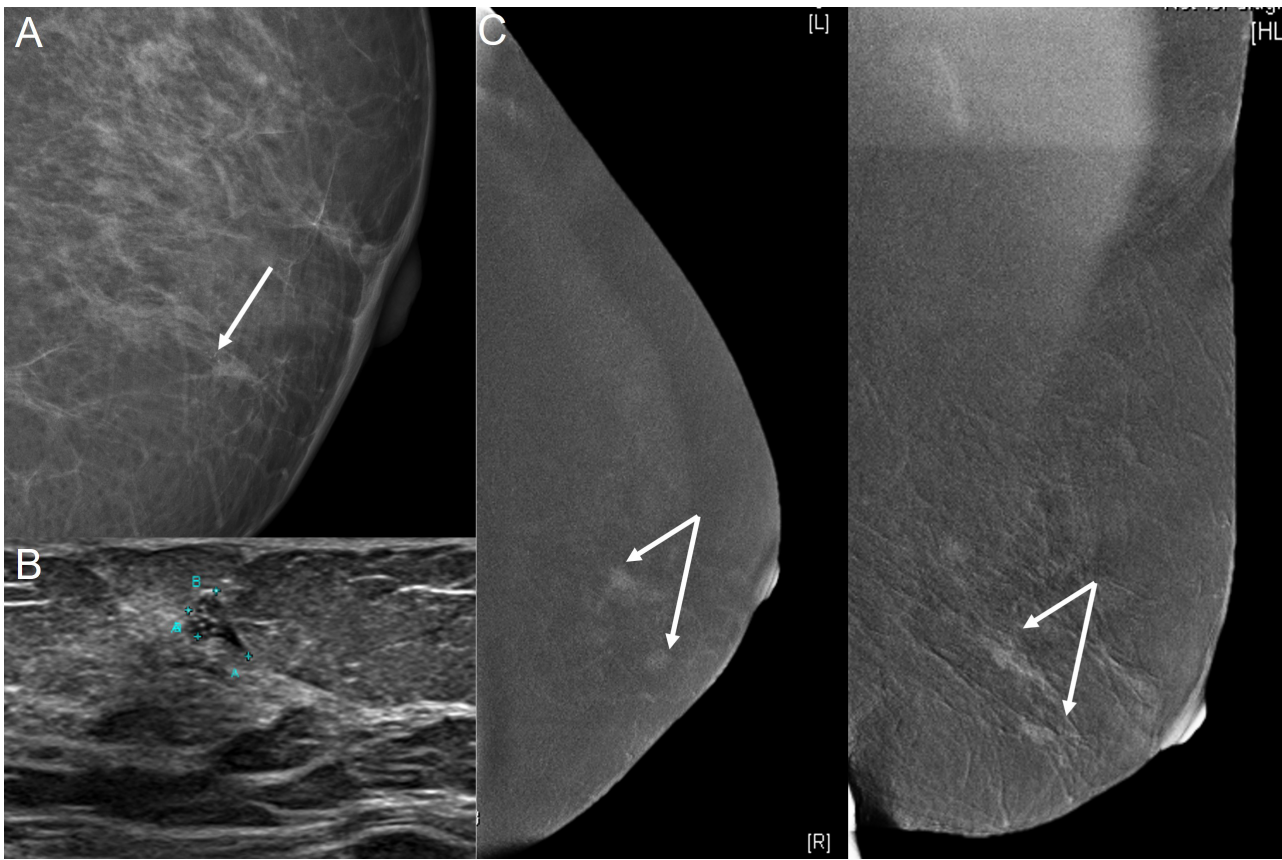

Figure 5. 64-year women with previous right mastectomy on routine surveillance left mammogram. (A) Magnified left mammogram shows a small tight group of microcalcifications (arrow) that was new compared to previous study; (B) Ultrasound shows a small, hypoechoic mass with internal calcifications (arrow) to correlate with mammographic finding; (C) Contrast enhanced mammogram images show clumped non-mass enhancement in the lower inner quadrant of the left breast (arrows). The extent of enhancement is much larger compared to that seen on mammogram and ultrasound. Final surgical histology proved $20 \mathrm{~mm}$ DCIS with $2 \mathrm{~mm}$ of invasive component. 
Biopsy of the lesion confirmed a high-grade DCIS with foci of microinvasion. Final surgical histology proved the tumor to be $20 \mathrm{~mm}$ with $2 \mathrm{~mm}$ of invasive component; the size better correlating with CEM than mammogram or ultrasound. We postulate that the non-calcified portion of cancer was better demonstrated on contrast study and CEM could have resulted in earlier detection of the recurrence.

\section{Literature review}

A few studies have already established the superiority of CEM over conventional mammogram for breast cancer screening [23] [24]. In a study by Sung et al. [24] of 904 women with higher-than-average risk, they reported $87 \%$ sensitivity and $93.7 \%$ specificity of CEM compared to only $50 \%$ sensitivity for mammogram. Nearly half the women in this study had a personal history of previous breast cancer. They reported CEM accuracy to be comparable to MRI in moderate to high-risk screening populations. No dedicated study has compared CEM with MRI in this group of population though. This opens scope for further research.

\subsection{Problem Solving in Equivocal Findings on Conventional Imaging}

The diagnostic accuracy of digital mammography is highly dependent on density of fibroglandular tissue, with dense parenchyma adversely affecting the sensitivity. Frequently, focal asymmetry and architectural distortion without any associated mass may present diagnostic challenges. Occasionally, ultrasound may have findings with borderline features and contribute to false positive biopsies. CEM can be used for further assessment in such patients and help the radiologist in assigning a more confident BIRADS category to the mammogram.

Case 1: A 53-year-old woman had a known history of left mastectomy for breast cancer. Routine surveillance right mammogram was essentially normal. Adjuvant right breast ultrasound detected a new $8 \mathrm{~mm}$ irregular hypoechoic mass at 12 o'clock with angular margins and associated subtle architectural distortion (Figure 6(A)). Ultrasound guided core biopsy of the mass revealed usual ductal hyperplasia without any evidence of malignancy. However, the patient was apprehensive and was keen for surgery, even requesting a mastectomy. CEM was performed as a problem-solving technique. CEM (Figure 6(B)) showed no enhancement in the right breast at 12 o'clock, favoring benign etiology. The patient was reassured and agreed for follow up. On serial imaging, the lesion remained stable over 2 years.

\section{Literature review}

Lalji et al. [25] reported CEM to be superior to mammography with an excellent problem-solving capability in patients recalled from screening program. CEM was shown to have higher sensitivity of up to $97 \%$ and specificity of up to $70 \%$ and improved diagnostic accuracy regardless of the reader's experience.

Lobbes et al. [26] prospectively analyzed the role of CEM in 113 women to address inconclusive findings on screening mammography. They reported $100 \%$ 


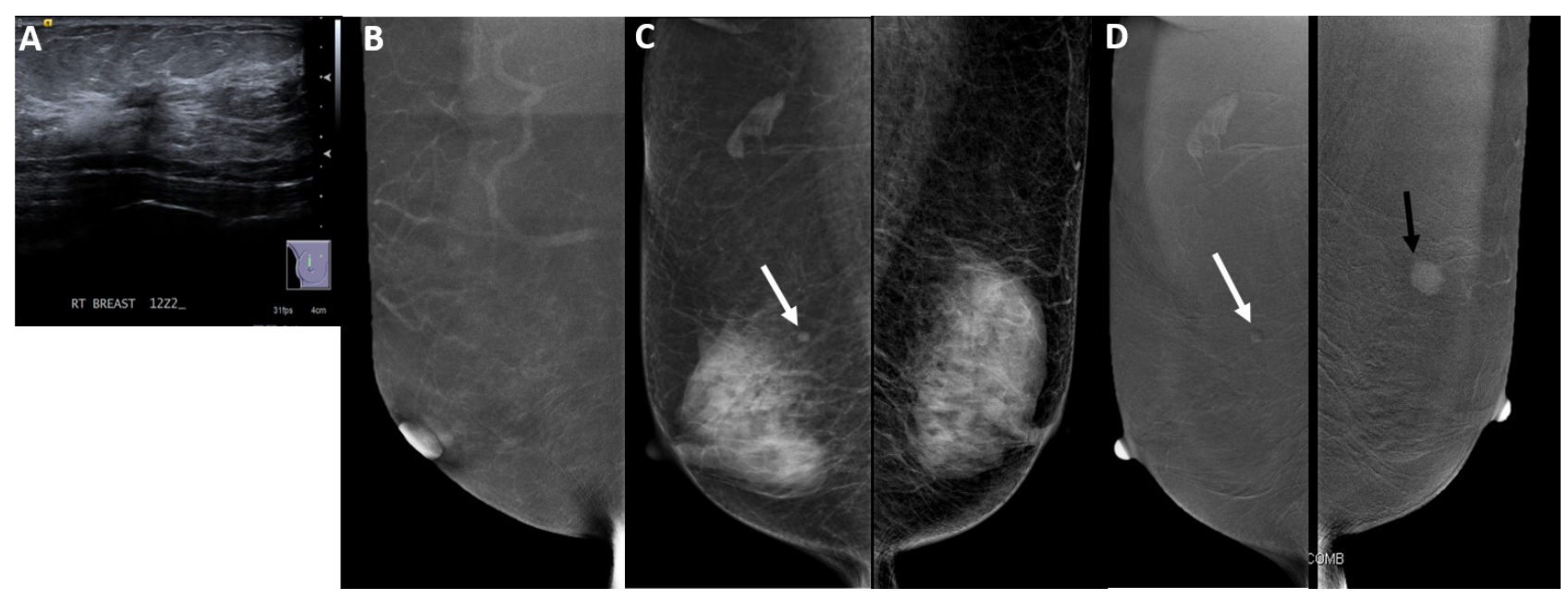

Figure 6. 53-year woman with previous left mastectomy on routine surveillance. Routine surveillance right mammogram was essentially normal (not shown) Adjuvant right breast ultrasound (A) shows a new subcentimeter, irregular, hypoechoic lesion with angular margins, architectural distortion and mixed posterior features at 12 o'clock location. Minimal internal vascularity is noted. Contrast enhanced mammogram image; (B) shows no enhancement in the right breast. Histology of right breast lesion was proven benign. Screening mammogram; $(C)$ in another 69-year high-risk woman shows extremely dense breast parenchyma. A sub-centimeter circumscribed round opacity in right retro-mammary fat (arrow) was noted; (D) Contrast enhanced mammogram images show no significant enhancement in the right breast lesion (white arrow). Incidentally an avidly enhancing mass was noted in the upper outer quadrant of the left breast (black arrow). Ultrasound (not shown) showed a small intra-mammary node in the right breast and an indeterminate lesion in the left breast to correlate with the enhancing mass. Histology of left breast mass was reported as invasive papillary carcinoma. It was a unifocal tumor as seen on CEM.

sensitivity as well as $100 \%$ Negative predictive values of CEM with statistically significant differences between mammogram and CEM. They reported better diagnostic accuracy of CEM even in average risk screening population recalled for assessment.

Houben et al. [27] retrospectively analyzed 839 women who underwent CEM after a screening recall. They found 70 CESM only lesions in 65 women; more than $50 \%$ of them were malignant. The most commonly detected benign abnormality on CEM was fibroadenoma. They reported CEM as a low risk, problem solving tool in screening recalls that might have significant clinical implications in patient's management.

\subsection{Screening in High-Risk Woman}

Women with lifetime risk of $>20 \%$ are grouped as high-risk population. The American Cancer Society (ACS) recommends annual screening with MRI and mammography in these women. Again, annual MRI screening may be not feasible or followed as recommended in many countries due to cost constraints. CEM may be utilized as an alternative tool to MRI in high-risk screening based on the current evidence suggesting comparable diagnostic performance of CEM over MRI with high negative predictive value [8] [12] [13] [15] [16].

Case 1: A 69-year-old woman with a strong family history of breast cancer in multiple relatives underwent screening mammogram. Mammogram (Figure 6(C)) showed dense breast parenchyma. A round, sub-centimeter, circumscribed opacity 
was observed in the right breast pre-pectoral fat. In view of the dense breast tissue and strong family history, she was offered additional screening with CEM. The right breast showed an intra-mammary lymph node. However, an avidly enhancing mass was detected in the left breast (Figure 6(D)). Targeted ultrasound showed an intra-mammary node in the right breast and a lobulated, indeterminate hypoechoic nodule in the left breast that was proven to be an in-situ $24 \mathrm{~mm}$ papillary carcinoma with solid and cribriform component; ER/PR positive.

\section{Literature review}

Sorin et al. [28] reported the usefulness of CEM in high-risk screening. They reported a significantly high, additional 13.1 cancers on CEM per 1000 intermediate risk women screened, compared to screening mammography. There was significantly higher sensitivity of CEM, up to $90.5 \%$, compared to mammogram which showed sensitivity of up to $52.4 \%$.

Jordana Phillips et al. [29] compared CEM versus MRI in high risk setting primarily evaluating in terms of patient preferences. They did prospective analysis of 43 patients in high risk setting and showed that $79 \%$ patients preferred CEM over MRI if the exams had equal sensitivity. Almost $89 \%$ would be comfortable receiving contrast as part of an annual screening test and about 97\% found the duration of CESM perfect for a screening test. They concluded that a high-risk population may prefer CEM as a screening tool, if ongoing trials demonstrate its results as being non-inferior to MRI.

\subsection{To Evaluate Cancers of Unknown Primary with Metastatic Axillary Lymphadenopathy}

MR imaging has already been established as the most sensitive modality for the detection of occult breast cancer in women who present with metastatic axillary adenopathy [30] [31] [32]. The identification of primary lesion is very important from the management perspective. The conventional treatment approach for occult breast cancer is mastectomy. However, if a primary lesion can be identified in a breast pre-operatively, the option of breast conservation may be offered to the patient.

CEM has shown similar sensitivity to MRI in detection of breast cancer [33] [34] [35] [36] [37] and may play an important role in such patients. There is no study currently showing the role of CEM in the detection of occult breast cancer. However, with ongoing improvements in CEM techniques, and better understanding and interpretation of images, CEM may be offered to patients as a primary tool where there is limited availability of MRI. Further research is warranted.

\section{Conclusions}

Multiple studies comparing CEM to MRI have shown CEM to have equivalent diagnostic efficiency with fewer false-positive results. Larger multicenter trials are expected to validate the use of CEM as an alternate to MRI in many indications. 
Distinct advantages of CEM include faster imaging and interpretation time, greater ease of scheduling the examination, lower cost, better patient comfort and lack of claustrophobia. Limitations of CEM include slightly increased radiation dose, contrast media reactions and inability to use this modality in patients with compromised renal function.

It is also important to remember that some benign lesions may also enhance on CEM, contributing to false-positive results. Hence, CEM is better interpreted in combination with morphological features of lesions on ultrasound. At this time, CEM may be considered as an option in cases where MRI is contraindicated or not available, e.g. in patients with MR incompatible cardiac pacemaker, claustrophobia, limited resources and cost issue.

\section{Teaching Points}

1) CEM is a new emerging modality in breast imaging that combines the advantages of mammograms and MRI;

2) Sensitivity of CEM is comparable to MRI;

3) Indications of CEM are nearly similar to that of MRI and at this time, CEM may be considered as an option in cases where MRI is contraindicated or not available;

4) CEM uses iodinated contrast similar to CT scan. It is contraindicated in patients with severe drug allergies, renal impairment and active asthma.

\section{Acknowledgements}

The authors would like to acknowledge Dr. Rishu Sangal for her assistance in reviewing the literature.

\section{Funding}

The cases were partly funded by the "Pitch for Fund Program" at Tan Tock Seng Hospital and "National Medical Research Council CSA grant (MOH-000279)".

\section{Conflicts of Interest}

The authors declare no conflicts of interest regarding the publication of this paper.

\section{References}

[1] Tabar, L., Fagerberg, G., Duffy, S.W., Day, N.E., et al. (1992) Update of the Swedish Two-County Program of Mammographic Screening for Breast Cancer. Radiologic Clinics of North America, 30, 187-210.

[2] Nystrom, L., Rutqvist, L.E., Wall, S., et al. (1993) Breast Cancer Screening with Mammography: Overview of Swedish Randomised Trials. The Lancet, 341, 973-978. https://doi.org/10.1016/0140-6736(93)91067-V

[3] Kuhl, C.K., Schmutzler, R.K., Leutner, C.C., Kempe, A., Wardelmann, E., Hocke, A., Maringa, M., Pfeifer, U., Krebs, D. and Schild, H.H. (2000) Breast MR Imaging Screening in 192 Women Proved or Suspected to Be Carriers of a Breast Cancer Susceptibility Gene: Preliminary Results. Radiology, 215, 267-279. 
https://doi.org/10.1148/radiology.215.1.r00ap01267

[4] Leach, M.O., Boggis, C.R., Dixon, A.K., et al. (2005) Screening with Magnetic Resonance Imaging and Mammography of a UK Population at High Familial Risk of Breast Cancer: A Prospective Multicentre Cohort Study (MARIBS). The Lancet, 365, 1769-1778. https://doi.org/10.1016/S0140-6736(05)66481-1

[5] Ciatto, S., Houssami, N., Bernardi, D., Caumo, F., Pellegrini, M., Brunelli, S., Tuttobene, P., Bricolo, P., Fantò, C., Valentini, M., Montemezzi, S. and Macaskill, P. (2013) Integration of 3D Digital Mammography with Tomosynthesis for Population Breast-Cancer Screening (STORM): A Prospective Comparison Study. The Lancet Oncology, 14, 583-589. https://doi.org/10.1016/S1470-2045(13)70134-7

[6] Heywang-Kobrunner, S.H., Bick, U., Bradley, W.G., Boné, B., Casselman, J., Coulthard, A., Fischer, U., Müller-Schimpfle, M., Oellinger, H., Patt, R., Teubner, J., Friedrich, M., Newstead, G., Holland, R., Schauer, A., Sickles, E.A., Tabar, L., Waisman, J. and Wernecke, K.D. (2001) International Investigation of Breast MRI: Results of a Multicentre Study (11 Sites) Concerning Diagnostic Parameters for Contrast-Enhanced MRI Based on 519 Histopathologically Correlated Lesions. European Radiology, 11, 531-546. https://doi.org/10.1007/s003300000745

[7] Heywang, S.H., Hahn, D., Schmidt, H., Krischke, I., Eiermann, W., Bassermann, R. and Lissner, J. (1986) MR Imaging of the Breast Using Gadolinium-DTPA. Journal of Computer Assisted Tomography, 10, 199-204. https://doi.org/10.1097/00004728-198603000-00005

[8] Fallenberg, E.M., Dromain, C., Diekmann, F., Engelken, F., Krohn, M., Singh, J.M., Ingold-Heppner, B., Winzer, K.J., Bick, U. and Renz, D.M. (2014) Contrast-Enhanced Spectral Mammography versus MRI: Initial Results in the Detection of Breast Cancer and Assessment of Tumour Size. European Radiology, 24, 256-264.

https://doi.org/10.1007/s00330-013-3007-7

[9] Mori, M., Akashi-Tanaka, S., Suzuki, S., Daniels, M.I., Watanabe, C., Hirose, M. and Nakamura, S. (2017) Diagnostic Accuracy of Contrast-Enhanced Spectral Mammography in Comparison to Conventional Full-Field Digital Mammography in a Population of Women with Dense Breasts. Breast Cancer, 24, 104-110.

https://doi.org/10.1007/s12282-016-0681-8

[10] Lee-Felker, S.A., Tekchandani, L., Thomas, M., Gupta, E., Andrews-Tang, D., Roth, A., Sayre, J. and Rahbar, G. (2017) Newly Diagnosed Breast Cancer: Comparison of Contrast-enhanced Spectral Mammography and Breast MR Imaging in the Evaluation of Extent of Disease. Radiology, 285, 389-400.

https://doi.org/10.1148/radiol.2017161592

[11] Cheung, Y.C., Juan, Y.H., Lo, Y.F., Lin, Y.-C., Yeh, C.-H. and Ueng, S.-H. (2020) Preoperative Assessment of Contrast-Enhanced Spectral Mammography of Diagnosed Breast Cancers after Sonographic Biopsy: Correlation to Contrast-Enhanced Magnetic Resonance Imaging and 5-Year Postoperative Follow-Up. Medicine, 99, e19024. https://doi.org/10.1097/MD.0000000000019024

[12] Dromain, C., Canale, S., Bidault, F., et al. (2011) Dual-Energy Contrast-Enhanced Digital Mammography: Initial Clinical Results. European Radiology, 21, 565-574.

[13] Jochelson, M.S., Dershaw, D.D., Sung, J.S., Heerdt, A.S., Thornton, C., Moskowitz, C.S., Ferrara, J. and Morris, E.A. (2013) Bilateral Contrast-Enhanced Dual-Energy Digital Mammography: Feasibility and Comparison with Conventional Digital Mammography and MR Imaging in Women with Known Breast Carcinoma. Radiology, 266, 743-751. https://doi.org/10.1148/radiol.12121084

[14] Fallenberg, E.M., Dromain, C., Diekmann, F., Engelken, F., Krohn, M., Singh, J.M., Ingold-Heppner, B., Winzer, K.J., Bick, U. and Renz, D.M. (2014) Contrast-Enhanced Spectral Mammography versus MRI: Initial Results in the Detection of Breast Can- 
cer and Assessment of Tumour Size. European Radiology, 24, 256-264. https://doi.org/10.1007/s00330-013-3007-7

[15] Łuczyńska, E., Heinze-Paluchowska, S., Hendrick, E., Dyczek, S., Ryś, J., Herman, K., Blecharz, P., and Jakubowicz, J. (2015) Comparison between Breast MRI and Contrast-Enhanced Spectral Mammography. Medical Science Monitor, 21, 1358-1367. https://doi.org/10.12659/MSM.893018

[16] Lewin, J. (2018) Comparison of Contrast-Enhanced Mammography and Contrast-Enhanced Breast MR Imaging. Magnetic Resonance Imaging Clinics of North America, 26, 259-263. https://doi.org/10.1016/j.mric.2017.12.005

[17] Hobbs, M.M., Taylor, D.B., Buzynski, S., et al. (2015) Contrast-Enhanced Spectral Mammography (CESM) and Contrast Enhanced MRI (CEMRI): Patient Preferences and Tolerance. Journal of Medical Imaging and Radiation Oncology, 59, 300-305. https://doi.org/10.1111/1754-9485.12296

[18] Lobbes, M.B., Prevos, R., Smidt, M., et al. (2013) The Role of Magnetic Resonance Imaging in Assessing Residual Disease and Pathologic Complete Response in Breast Cancer Patients Receiving Neoadjuvant Chemotherapy: A Systematic Review. Insights into Imaging, 4, 163-75. https://doi.org/10.1007/s13244-013-0219-y

[19] Barra, F.R., Sobrinho, A.B., Barra, R.R., et al. (2018) Contrast-Enhanced Mammography (CEM) for Detecting Residual Disease after Neoadjuvant Chemotherapy: A Comparison with Breast Magnetic Resonance Imaging (MRI). BioMed Research International, 2018, Article ID: 8531916. https://doi.org/10.1155/2018/8531916

[20] Iotti, V., Ravaioli, S., Vacondio, R., et al. (2017) Contrast-Enhanced Spectral Mammography in Neoadjuvant Chemotherapy Monitoring: A Comparison with Breast Magnetic Resonance Imaging. Breast Cancer Research, 19, Article No. 106. https://doi.org/10.1186/s13058-017-0899-1

[21] Hetal, M.H., Mansour, S.M., Ahmed, H.A., et al. (2019) Role of Contrast Enhanced Spectral Mammography in the Evaluation of Post-Operative Breast Cancer. Clinical Radiology, 74, 771-781. https://doi.org/10.1016/j.crad.2019.06.002

[22] Swinnen, J., Keupers, M., Soens, J., et al. (2018) Breast Imaging Surveillance after Curative Treatment for Primary Non-Metastasised Breast Cancer in Non-High-Risk Women: A Systematic Review. Insights into Imaging, 9, 961-970. https://doi.org/10.1007/s13244-018-0667-5

[23] Kim, G., Phillips, J., Cole, E., et al. (2019) Comparison of Contrast-Enhanced Mammography with Conventional Digital Mammography in Breast Cancer Screening: A Pilot Study. Journal of the American College of Radiology, 16, 1456-1463. https://doi.org/10.1016/j.jacr.2019.04.007

[24] Sung, J.S., Lebron, L., Keating, D., et al. (2019) Performance of Dual-Energy Contrast-Enhanced Digital Mammography for Screening Women at Increased Risk of Breast Cancer. Radiology, 293, 81-88. https://doi.org/10.1148/radiol.2019182660

[25] Lalji, U.C., Houben, I.P., Prevos, R., et al. (2016) Contrast-Enhanced Spectral Mammography in Recalls from the Dutch Breast Cancer Screening Program: Validation of Results in a Large Multireader, Multicase Study. European Radiology, 26, 4371-4379. https://doi.org/10.1007/s00330-016-4336-0

[26] Lobbes, M.B., Lalji, U., Houwers, J., et al. (2014) Contrast-Enhanced Spectralmammography in Patients Referred from the Breast Cancer Screening Programme. European Radiology, 24, 1668-1676. https://doi.org/10.1007/s00330-014-3154-5

[27] Houben, I.P.L, Van de Voorde, P., Jeukens, C.R.L.P.N., et al. (2017) Contrast-Enhanced Spectral Mammography as Work-Up Tool in Patients Recalled from Breast Cancer 
Screening Has Low Risks and Might Hold Clinical Benefits. European Journal of Radiology, 94, 31-37. https://doi.org/10.1016/j.ejrad.2017.07.004

[28] Sorin, V., Yagil, Y., Yosepovich, A., et al. (2018) Contrast-Enhanced Spectral Mammography in Women with Intermediate Breast Cancer Risk and Dense Breasts. American Journal of Roentgenology, 211, W267-W274.

https://doi.org/10.2214/AJR.17.19355

[29] Phillips, J., Miller, M.M., Mehta, T.S., et al. (2017) Contrast-Enhanced Spectral Mammography (CESM) versus MRI in the High-Risk Screening Setting: Patient Preferences and Attitudes. Clinical Imaging, 42, 193-197.

https://doi.org/10.1016/j.clinimag.2016.12.011

[30] Orel, S.G., Weinstein, S.P., Schnall, M.D., et al. (1999) Breast MR Imaging in Patients with Axillary Node Metastases and Unknown Primary Malignancy. Radiology, 212, 543-549. https://doi.org/10.1148/radiology.212.2.r99au40543

[31] Buchanan, C.L., Morris, E.A., Doru, P.L., Borgen, P.I. and Van Zee, K.J. (2005) Utility of Breast Magnetic Resonance Imaging in Patients with Occult Primary Breast Cancer. Annals of Surgical Oncology, 12, 1045-1053.

https://doi.org/10.1245/ASO.2005.03.520

[32] Morris, E.A., Liberman, L., Ballon, D.J., et al. (2003) MRI of Occult Breast Carcinoma in a High Risk Population. American Journal of Roentgenology, 181, 619-626. https://doi.org/10.2214/ajr.181.3.1810619

[33] Zhu, X., Huang, J.M., Zhang, K., et al. (2018) Diagnostic Value of Contrast-Enhanced Spectral Mammography for Screening Breast Cancer: Systematic Review and Meta-Analysis. Clinical Breast Cancer, 18, E985-E995.

https://doi.org/10.1016/j.clbc.2018.06.003

[34] Tagliafico, A.S., Bignotti, B., Rossi, F., Signori, A., Sormani M.P., Valdora, F., Calabrese, M. and Houssami, N. (2016) Diagnostic Performance of Contrast-Enhanced Spectral Mammography: Systematic Review and Meta-Analysis. Breast, 28, 9-13. https://doi.org/10.1016/j.breast.2016.04.008

[35] Suter, M.B., Pesapane, F., Agazzi, G.M., Gagliardi, T., Nigro, O. Bozzini, A., Priolo, F., Penco, S., Cassano, E., Chini, C. and Squizzato, A. (2020) Diagnostic Accuracy of Contrast-Enhanced Spectral Mammography for Breast Lesions: A Systematic Review and Meta-Analysis. Breast, 53, 8-17. https://doi.org/10.1016/j.breast.2020.06.005

[36] Xiang, W., Rao, H. and Zhou, L. (2020) A Meta-Analysis of Contrast-Enhanced Spectral Mammography versus MRI in the Diagnosis of Breast Cancer. Thoracic Cancer, 11, 1423-1432. https://doi.org/10.1111/1759-7714.13400

[37] Sogani, J., Mango, V.L., Keating, D., Sung, J.S. and Jochelson, M.S. (2021) ContrastEnhanced Mammography: Past, Present, and Future. Clinical Imaging, 69, 269-279. https://doi.org/10.1016/j.clinimag.2020.09.003 